\title{
Active control of a balanced two-stage pendulum vibration isolation system and its application to laser interferometric gravity wave detectors
}

\author{
P. J. Veitch, a) N. A. Robertson, C. A. Cantley, and J. Hough \\ Department of Physics and Astronomy, University of Glasgow, Glasgow G12 $8 Q Q$, Scotland
}

(Received 28 September 1992; accepted for publication 7 January 1993)

\begin{abstract}
The investigation of the servo control of the position of the bottom mass in a balanced two-stage pendulum vibration isolation system is reported. Experimental results for a simple prototype system and predictions based on a model presented in this paper are in good agreement. The application of such a system to a high-sensitivity laser interferometric gravity wave detector is discussed.
\end{abstract}

\section{INTRODUCTION}

The isolation of test masses from spurious mechanical vibrations is of growing importance in high-precision experiments, particularly in laser interferometric gravitational wave detectors. Such detectors rely in principle on sensing the change, induced by a gravitational wave, in the relative separation of a number of almost freely suspended test masses. In its simplest form a laser interferometric detector consists of three test masses suspended at three corners of a square to form two perpendicular arms or baselines; and the relative length of the two arms is monitored using laser interferometry between very-highreflectance mirrors attached to these masses. ${ }^{1}$

To maximize the sensitivity of the interferometer the test masses must be isolated from external influences. Thus, the interferometer is housed in a vacuum enclosure to minimize test mass motion produced by acoustic noise, and optical path-length changes due to refractive index fluctuations. The test masses are also isolated from highfrequency ( $>$ few $\mathrm{Hz}$ ) seismic and environmental vibrations using vibration isolation systems which typically incorporate lead/rubber vibration isolation stacks and pendulums. ${ }^{2}$ To ensure that Nyquist noise in these systems does not limit the detector sensitivity, the parts of the suspension which directly support each of the test masses must have low mechanical losses. However, the seismic excitation of the concomitant high- $Q$ mechanical resonances may result in large low-frequency motion of the test masses. To allow the interferometer to operate with maximum sensitivity the low-frequency motion must be reduced to $<10^{-12} m_{\mathrm{rms}} \cdot{ }^{1}$ This can be achieved by actively damping the resonances using auxiliary feedback systems, and using the output from the interferometer to servo control the relative axial positions of the test masses.

The interferometer output will also contain any signals produced by a passing gravity wave. Signals at frequencies above the unity gain frequency of the servo will be directly observable at the interferometer output while signals at frequencies below the unity gain frequency will be best monitored in the feedback signal used to control the relative axial positions of the test masses. Contributions due to

\footnotetext{
a) Now at Department of Physics and Mathematical Physics, University of Adelaide, Australia.
}

laser shot noise, frequency and intensity noise, and servo electronic noise will also be contained in the signals being examined for gravity waves, and will limit the detector sensitivity.

There are several proposed methods for suspending the test masses in high-sensitivity, long baseline laser interferometric detectors. ${ }^{3-5}$ In this article we briefly describe one possible design, ${ }^{3}$ shown schematically in Fig. 1, and report results of experimental testing and modeling of a simplified prototype version shown in Fig. 2. The prototype suspension was designed to facilitate the investigation of the servo control of the two-stage balanced pendulum. As will be discussed in Sec. II, it has several features that could not be used in a high-sensitivity interferometer but which do not affect the testing of the servo control.

In the design shown in Fig. 1, care is taken to minimize the number of electromechanical systems acting directly on the test mass as these are potential sources of additional noise. This is accomplished by suspending each test mass using a balanced two-stage pendulum in which the test mass and a reaction mass are suspended from the same intermediate mass, and applying most of the feedback signals to the intermediate and reaction pendulum masses. In particular, the orientation of the test mass and the damping of its pendulum motion are achieved by applying correction forces to the intermediate and reaction masses. In the prototype suspension, however, it was decided for reasons of experimental simplicity to apply the orientation control forces directly to the test mass.

There are several other reasons for choosing a balanced two-stage configuration. First, the vertical isolation provided by the two stages, together with that provided by the multistage vibration isolation stack $^{6}$ which supports the pendulum (see Fig. 1), ensures that axial motion produced by vertical-to-axial cross coupling should be sufficiently small. The balanced final stage provides a well-isolated platform (the reaction mass) from which to apply feedback forces to the test mass (see below), thereby preventing the coupling of spurious mechanical vibrations to the test mass via the actuator. It also limits the excitation of internal resonances in the stack top-plate by the feedback and helps to maintain the alignment of the test-mass actuator as the orientation of the test mass is adjusted.

The axial position of the test mass is controlled using split feedback ${ }^{7,8}$ in which the large-amplitude, low- 


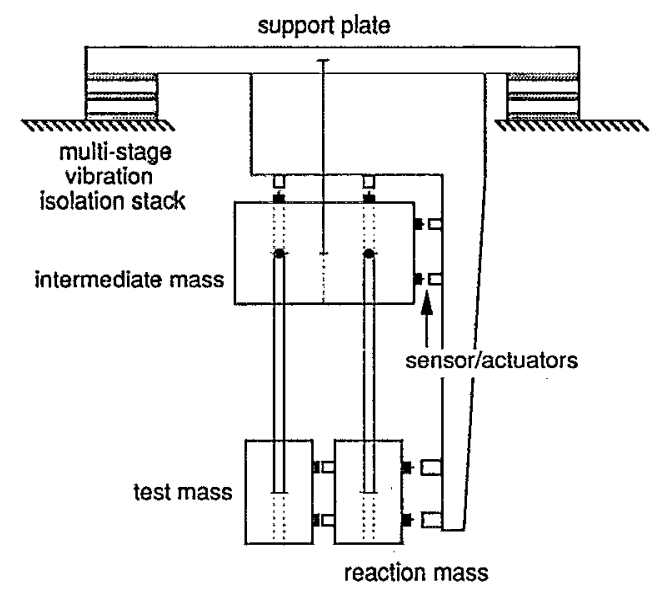

FIG. 1. Schematic diagram of the test mass suspension for a highsensitivity interferometer. Both the test mass and reaction mass are suspended from the intermediate mass using a two-loop (four-wire) pendulum suspension. The intermediate mass is suspended using a single loop. Test mass orientation is controlled by acting on the intermediate mass, and its axial position is controlled using split feedback as discussed in the text.

frequency signals are fed back to the intermediate mass and only the smaller-amplitude, higher-frequency signals are fed back to the test mass, as shown in Fig. 2. This may reduce the effects of frequency up-conversion of large lowfrequency fluctuations by nonlinearities in the electromechanical drive systems. Split feedback systems may be characterized according to the relative values of the "crossover" frequency and the frequencies of the pendulum normal modes, where the cross-over frequency is the frequency which separates the region in which servo control is dominated by the feedback to the intermediate mass (below the cross-over) from that in which it is dominated by the feedback to the test mass (above the cross-over).

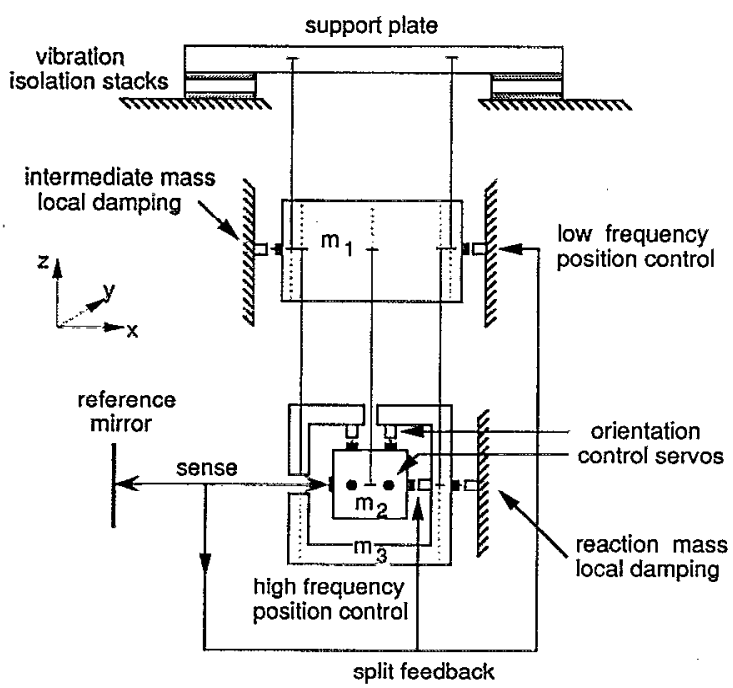

FIG. 2. Schematic diagram of the prototype balanced two-stage pendulum. The masses and uncoupled resonant frequencies of the pendulums are intermediate mass, $11.0 \mathrm{~kg}$ and $5.9 \mathrm{~s}^{-1}$; test mass, $3.3 \mathrm{~kg}$ and $4.7 \mathrm{~s}^{-1}$; reaction mass, $2.3 \mathrm{~kg}$ and $4.7 \mathrm{~s}^{-1}$.
The system discussed here has a cross-over frequency just below the pendulum normal mode frequencies.

The investigation of an alternative suspension system has recently been reported by Stephens et al. ${ }^{9}$ That design used a nested two-stage pendulum, in which the test mass was enclosed by a concentric cylindrical intermediate mass. The damping of the pendulum modes was achieved by sensing the motion of, and feeding back to, the intermediate mass, and the position of the test mass was controlled by using the intermediate mass as a reaction mass from which to apply (all of the) correction forces to the test mass.

\section{DESCRIPTION OF EXPERIMENTAL PENDULUM SUSPENSION}

A schematic diagram of the system used to investigate the split feedback servo control of the balanced two-stage pendulum ${ }^{7,8}$ is shown in Fig. 2. The intermediate mass is suspended by two wire loops from a support plate that is isolated from the ground by a two-stage metal and rubber vibration isolation stack. The test mass is suspended from the intermediate mass by a single loop of wire and enclosed by a box-shaped reaction mass that is suspended from the intermediate mass using two wire loops. This arrangement enabled the test mass's orientation in tilt (about the $y$ axis) and rotation (about the $z$ axis) to be conveniently controlled using shadow-position-sensor/magnetic-actuator assemblies ${ }^{10}$ mounted on the reaction mass. As indicated in Sec. I this arrangement may not be suitable for use in a high-sensitivity interferometer, because it could require the application of large forces by the test/reaction mass actuators which might result in significant nonlinear production of noise.

The shadow-position-sensor assemblies consist of a flag which is mounted on the sensed surface, the test mass in this case, and is free to move between a light-emitting diode (LED) and photodiode which are mounted on the reference surface, the reaction mass. Relative motion modulates the light incident on the photodiode, thereby producing a signal proportional to the relative displacement of the test and reaction masses. After suitable amplification and filtering, this signal can be fed back to a coil mounted on the reaction mass, which applies a force to a magnet mounted on the test mass.

The $Q$ 's of the pendulum normal modes are damped by sensing the position of the intermediate and reaction masses relative to a local ground reference and applying correction forces which are proportional to velocity (at low frequencies) using shadow-sensor/magnetic-actuator assemblies described above. This type of servo control will hereafter be referred to as local damping. In this particular case it is referred to the local ground, but it may in general be referred to any surface.

The coupling of high-frequency noise into these masses due to vibration of the reaction surface is minimized by using an actuator which produces a force that has a minimal dependence on the separation of the reaction surface and servoed mass (i.e., has minimal spring constant). The coupling of noise into the servoed mass due to vibration of 


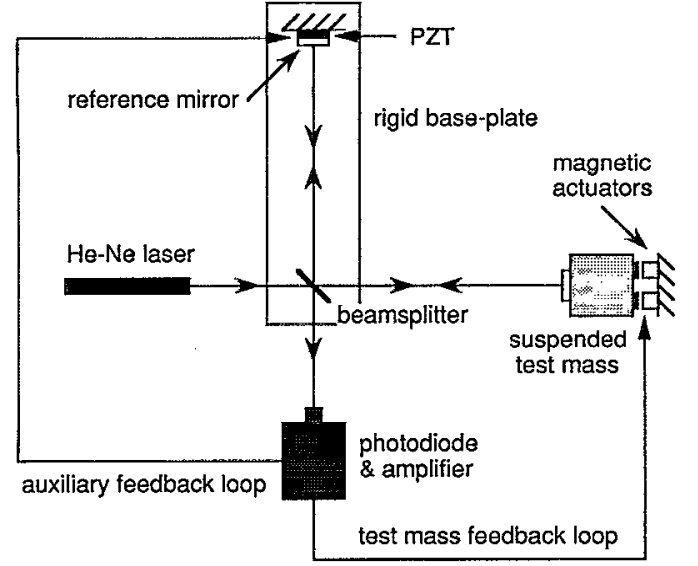

FIG. 3. Layout of the Michelson interferometer used to monitor the test mass position.

the sensor reference surface is minimized by "rolling-off" the servo gain at frequencies above the pendulum normal mode frequencies. Since these servos require only relatively low loop gain to critically damp the normal modes, they can have small bandwidths and thus the velocity of the servoed mass will be determined by that of the reference point in only a small band of frequencies around the normal mode frequencies. Also, since the electronics does not need to be dc coupled, the mass position will be less affected by dc drift and creep in the reference and the normal mode frequencies will remain essentially unchanged.

However, even with these precautions, the groundreferred local damping configuration described above would probably not be acceptable in a high-sensitivity interferometer as it could lead to the coupling of excessive levels of (high frequency) noise into the intermediate and reaction masses due to the seismic vibration of the reference/reaction surface. In such an interferometer a stiff extension of the vibration isolated top plate could provide the reference and reaction element for the intermediate mass and reaction mass actuators as shown in Fig. 1.

The position of the test mass in the axial ( $x$ axis) direction is sensed with respect to a reference point using a Michelson interferometer as shown in Fig. 3. The reference point used here is a mirror mounted on the ground. In a gravitational wave detector, however, the reference would be another suspended test mass. The signal from the photodiode is amplified and filtered to allow the low-frequency feedback signals to be fed back to the intermediate mass, and the higher frequency signals to be fed back to the test mass, as described in Scc. $I$. The intermediate mass feedback force is applied using coils mounted on a frame attached to the ground. As above, this arrangement would not be acceptable in a high-sensitivity interferometer because residual spring constant associated with the magnetic force might couple too much seismic noise into the movement of the test mass. This difficulty may be alleviated by the use of the stiff extension from the stack top plate, mentioned above, as a mounting point for the actuator coils.

An auxiliary feedback loop, in which some of the output signal is applied to a piezo-electric transducer (PZT) (a)

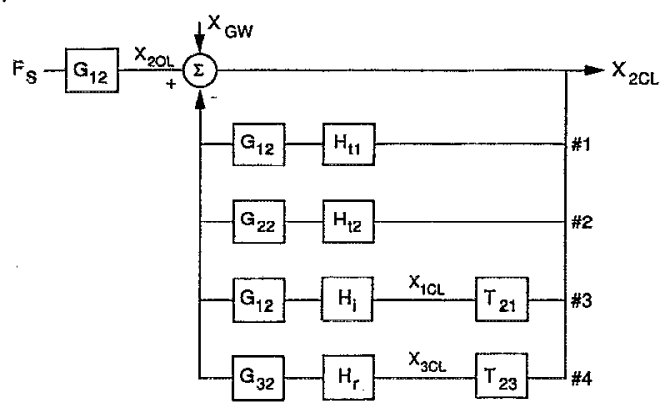

(b)
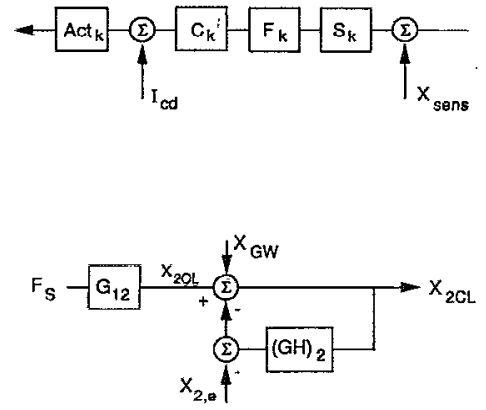

FIG. 4. (a) Servo block diagram used to determine the closed-loop motion of the test mass, $X_{2 \mathrm{CL}}$. Here $X_{G W}$ represents the motion of the test mass due to the action of a gravity wave. All other symbols are defined in the Appendix. (b) Elements contained in each feedback block $H_{k}$. Each block is comprised of a sensor $\left(S_{k}\right)$, an amplifier/filter $\left(F_{k}\right)$, a current driver $\left(C_{k}\right)$, and an actuator $\left(A c t_{k}\right)$. Here $X_{\text {sens }}$ represents the equivalent input displacement noise of the sensor due to both its own noise and to nonservo noise such as laser shot noise, and intensity and frequency noise. Here $I_{c d}$ represents the equivalent output current noise added by the current driver, which in certain experimental circumstances may be significant. In practice, $S_{t 1}$ would be the same physical sensor as $S_{t 2}$, and $C_{i}$ and $A c t_{i}$ would probably be the same physical devices as $C_{t 1}$ and $A c t_{t 1}$. (c) Condensed servo block diagram. Here $X_{2, e}$ represents the equivalent openloop test mass displacement produced by the noise sources in (b).

mounted between the rigid baseplate and the reference mirror, is used to help acquire servo control by effectively increasing the servo bandwidth and dynamic range when the initial motion of the pendulum is large. This makes it easier for the other feedback loops to acquire control, after which the gain of the auxiliary loop can be reduced to zero. The PZT also provides a useful means of injecting displacement noise of known amplitude into the feedback loop to facilitate measurement of the loop gain.

\section{MODELING}

The servo block diagram shown in Fig. 4(a) can be used to model the system. As shown in Fig. 4(b), each of the feedback blocks $H_{t 1}, H_{2}, H_{i}$, and $H_{r}$ represents an electromechanical chain consisting of a position sensor, an amplifier/filter, a current driver, and an actuator. To simplify the model we have ignored the presence of the vibration isolation stack. This should not compromise the model's usefulness for estimating the residual low-frequency motion, however, as conventional stacks do not attenuate 
low-frequency seismic vibrations significantly. The effect of nonseismic noise on the control of the test mass position can also be considered by incorporating noise added by the servo as represented by $X_{\text {sens }}$ and $I_{c d}$ in Fig. 4(b). All other symbols are defined in the Appendix.

The split feedback of the interferometer signal to the intermediate mass and test mass is modeled by feedback paths No. 1 and No. 2, respectively. The feedback blocks $H_{t 1}$ and $H_{t 2}$ transform the interferometer signal, which contains information about the relative arm lengths, or, in this case, the position of our test mass, to give the feedback forces applied to the intermediate and test masses. The response of the test mass to these forces is given by $G_{12}$ and $G_{22}$.

The effect of the intermediate mass local damping on the motion of the test mass is modeled by feedback path No. 3. The closed-loop motion of the intermediate mass can be deduced from the closed-loop motion of the test mass using $T_{21}$. It is detected by the local damping sensor contained in $H_{i}$, amplified and filtered, and a correction force applied to the intermediate mass. The effect of this force on the test mass motion is given by $G_{12}$. Similarly, the effect of the reaction mass local damping on the motion of the test mass is modeled by feedback path No. 4 , in which the closed-loop motion of the reaction mass is deduced from the closed-loop motion of the test mass using $T_{23}$. The local damping sensor in $H_{r}$ detects this motion, a feedback force is applied to the reaction mass, and the resulting test mass motion can be determined using $G_{32}$.

\section{A. Servo loop stability}

The block diagram in Fig. 4(a) can be condensed to that shown in Fig. $4(\mathrm{c})$, where the loop gain $(G H)_{2}$, is given by

$$
(G H)_{2}=G_{12} H_{t 1}+G_{22} H_{22}+G_{12} H_{i} T_{21}+G_{32} H_{r} T_{23} .
$$

Alternately, combining the equation for the closed-loop motion of the test mass due to seismic noise, $X_{2 C L}$, that can be obtained from Fig. 4(c),

$$
X_{2 C L}=\frac{G_{12} F_{s}}{1+(G H)_{2}}
$$

with the expressions for $X_{2 C L}$ and $G_{12}$ in the Appendix gives

$$
(G H)_{2}=\frac{e_{33} \operatorname{det}_{f b}-\left(e_{33}+H_{r}\right) \operatorname{det}_{n f}}{\left(e_{33}+H_{r}\right) \operatorname{det}_{n f}} .
$$

The characteristic equation, $1+(G H)_{2}=0$, which is used to check loop stability, is then given, as would be expected from eigenvalue analysis, by $\operatorname{det}_{f b}=0$.

There are two conditions which must be satisfied for the servo loop to be stable. First, at the frequency at which the magnitude of the loop gain passes through $0 \mathrm{~dB}$ (the unity gain frequency), the phase of the loop gain must be $<\pi$, and preferably be $\sim 2 \pi / 3$ to ensure acceptable transient response. Since the loop gain is dominated by the feedback to the test mass at high frequencies, this condition is equivalent to the requirement that the gain of $G_{22} H_{l}$ should not decrease faster than $40 \mathrm{~dB} /$ decade (averaged

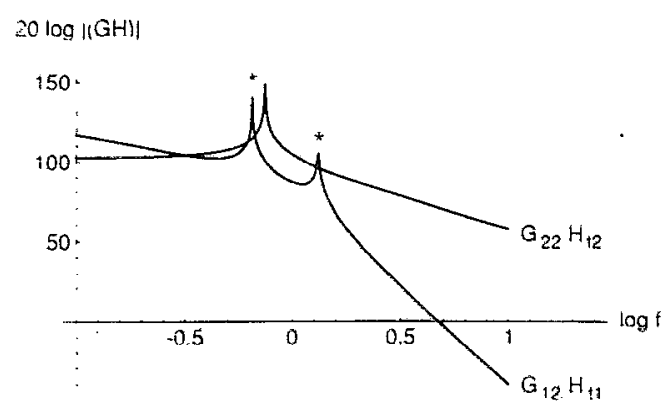

FIG. 5. Graph of the magnitudes of the split-feedback to the intermediate and test masses, $\left|G_{12} H_{t 1}\right|$ and $\left|G_{22} H_{t}\right|$. Note the additional crossovers at "*" due to the high $Q$ of the normal modes. The feedback parameters used to calculate this graph are the same as those used to calculate Fig. 7.

over about a decade) at the unity gain frequency. As the gain of $G_{22}$ is decreasing at $40 \mathrm{~dB} /$ decade, this requirement can only be satisfied if the gain of $H_{12}$ is increasing at the unity gain frequency. That is, $H_{t 2}$ should behave as a highpass filter or differentiator in the region of the unity gain frequency.

Second, at the cross-over frequency, the phase difference between the loop gain of the low-frequency feedback via the intermediate mass $\left(G_{12} H_{t 1}\right)$ and the loop gain of the feedback directly to the test mass $\left(G_{22} H_{i 2}\right)$ must be $<\pi$, and preferably be $\sim 2 \pi / 3$. That is, the magnitudes of these loop gains must not be changing relative to each other at a rate greater than $40 \mathrm{~dB} /$ decade. For a servo having a crossover frequency well below low- $Q$ pendulum normal modes, this condition is equivalent to the requirement that the gain of $H_{t 1}$ relative to the gain of $H_{22}$ (i.e., $\left|H_{t 1} / H_{22}\right|$ ) should not decrease faster than $40 \mathrm{~dB} /$ decade at the cross-over frequency. High- $Q$ normal modes, however, will result in additional incorrectly phased cross-overs, as shown in Fig. 5 , due to the difference between $G_{12}$ and $G_{22}$, and thus lead to instability. This instability can be removed by using local damping to decrease the $Q$ 's of the normal modes. It may not be possible to completely remove the effects of these normal modes, however, as the phase lag produced by the modes may result in an underdamped transient response of the servo. As would be expected and will be demonstrated in Sec. IV, the use of local damping to achieve stability becomes even more crucial as the crossover frequency approaches the normal modes.

\section{B. Closed-loop motion}

From Fig. 4(c), the total closed-loop motion of the test mass is given by

$$
X_{2 C L \text { (total) }}=\frac{X_{G W}+\left(G_{12} F_{s}+X_{2, c}\right)}{1+(G H)_{2}},
$$

where $X_{G W}$ represents the open-loop displacement due to a gravity wave (the signal) and $G_{12} F_{s}+X_{2, e}$ represents the open-loop displacement due to seismic noise and generalized electronic (nonseismic) noise.

It is convenient to divide the contribution due to noise into two parts: the relatively large-amplitude, lowfrequency $(<\sim 10 \mathrm{~Hz})$ motion due mostly to seismic 


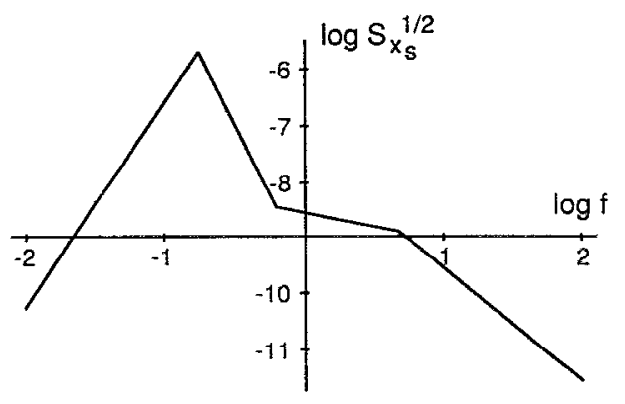

FIG. 6. Spectrum of seismic displacement noise assumed in the calculation of the rms residual displacement. This spectrum can be generated using $1.5 \times 10^{-3} f^{3.7} \mathrm{~m} / \mathrm{Hz}^{1 / 2}$ for $f<0.171 \mathrm{~Hz}, 3.8 \times 10^{-10} / f^{4.9}$ for 0.171 $\mathrm{Hz}<f<0.636 \mathrm{~Hz}, 2.8 \times 10^{-9} / f^{0.49}$ for $0.636 \mathrm{~Hz}<f<4.59 \mathrm{~Hz}$, and 2.8 $\times 10^{-8} / f^{2.0}$ for $f>4.59 \mathrm{~Hz}$.

noise, and the much smaller-amplitude, high-frequency $(>100 \mathrm{~Hz})$ motion, due mostly to nonseismic noise, which competes directly with the gravity wave signal. The main effect of the low-frequency motion is to disrupt the destructive interference of the light returning from each of the interferometer arms, thereby increasing the effect of other noise sources such as laser intensity noise and thus decreasing the interferometer sensitivity. The effect of these, and other (e.g., laser shot noise), non-servo-electronic noise sources can, in principle, be included in the equivalent input displacement noise of the sensor, $X_{\text {sens }}$, and thus in $X_{2, e}$

For a "properly designed" servo in which the openloop high-frequency noise is dominated by the noise at the "front end", the sensitivity of the interferometer is just given by $X_{2, e}$. To minimize the effect of gain changes and any "back-end" noise [e.g., current driver noise as represented by $I_{c d}$ in Fig. 4(b)], the gravity wave signal is best monitored at the front end (sensor output) for frequencies above the unity gain frequency, and at the feedback point (current driver output) for frequencies below the unity gain frequency.

A useful measure of the residual low-frequency motion, the rms motion of the test mass, $X_{2 \text { rms }}$ is given by

$$
X_{2 \mathrm{rms}}=\left[\int_{0}^{\infty} \frac{\left|G_{12}\right|^{2} S_{F_{s}}}{\left|1+(G H)_{2}\right|^{2}} d f\right]^{1 / 2},
$$

where $S_{F_{s}}$, the spectral density of force noise applied to the intermediate mass due to the seismic motion of the pendulum support point, is given by

$$
S_{F_{s}}(\omega)=\left|M \omega_{1}^{2}+j m_{1} \omega_{1} \omega / Q_{1}\right|^{2} S_{X_{s}}(\omega) .
$$

To enable evaluation of $X_{2 \mathrm{rms}}$ we assumed the seismic noise spectrum shown in Fig. 6, which is similar to that measured recently at JILA. ${ }^{11}$

\section{RESULTS}

The transfer functions of the feedback elements used in the experimental test of the split feedback system ${ }^{8}$ shown in Fig. 2 are given by

$$
\begin{gathered}
H_{t 1(\exp )}(s)=k_{1} /\left(\left(1+s \tau_{11}\right)\left(1+s \tau_{12}\right)\left(1+s \tau_{13}\right)\left(1+s \tau_{14}\right)^{2}\right), \\
H_{i 2(\exp )}(s)=k_{2}\left(1+s \tau_{21}\right) /\left(\left(1+s \tau_{22}\right)\left(1+s \tau_{23}\right)^{2}\right), \\
H_{i(\exp )}(s)=k_{i} s \tau_{i 1} /\left(\left(1+s \tau_{i 1}\right)\left(1+s \tau_{i 2}\right)\left(1+s \tau_{i 3}\right)\right) \\
H_{r(\exp )}(s)=k_{r}\left(1+s \tau_{r 1}\right) /\left(\left(1+s \tau_{r 2}\right)^{2}\left(1+s \tau_{r 3}\right)\right)
\end{gathered}
$$

where

$$
\begin{aligned}
& k_{1}=4.4 \times 10^{13} \mathrm{~N} / \mathrm{m}, \quad \tau_{11}=2.9 \times 10^{5} \mathrm{~s}, \\
& \tau_{12}=2 \mathrm{~s}, \quad \tau_{13}=2.2 \times 10^{-14} \mathrm{~s}, \quad \tau_{14}=4.8 \times 10^{-5} \mathrm{~s}, \\
& k_{2}=1.0 \times 10^{7} \mathrm{~N} / \mathrm{m}, \quad \tau_{21}=2.2 \times 10^{-3} \mathrm{~s} \\
& \tau_{22}=2.2 \times 10^{-4} \mathrm{~s}, \quad \tau_{23}=4.8 \times 10^{-5} \mathrm{~s} \\
& k_{i}=1.6 \times 10^{3} \mathrm{~N} / \mathrm{m}, \quad \tau_{i 1}=1.5 \times 10^{-2} \mathrm{~s} \\
& \tau_{i 2}=1.0 \times 10^{-2} \mathrm{~s}, \quad \tau_{i 3}=4.7 \times 10^{-3} \mathrm{~s} \\
& k_{r}=1.0 \times 10^{2} \mathrm{~N} / \mathrm{m}, \quad \tau_{r 1}=4.7 \times 10^{-2} \mathrm{~s} \\
& \tau_{r 2}=4.7 \times 10^{-3} \mathrm{~s}, \quad \tau_{r 3}=4.7 \times 10^{-4} \mathrm{~s} .
\end{aligned}
$$

Since some of the filters in the experimental transfer functions were only concerned with reducing the highfrequency gain so as to accommodate the finite amplifier bandwidths and to prevent the excitation of spurious resonances, the modeling can be simplified by using

$$
\begin{aligned}
& H_{t 1}(s)=k_{1} /\left(\left(1+s \tau_{11}\right)\left(1+s \tau_{12}\right)\right), \quad H_{n}(s)=k_{2}\left(1+s \tau_{21}\right), \\
& H_{i}(s)=\frac{k_{i} s \tau_{i 1}}{\left(1+s \tau_{i 1}\right)\left(1+s \tau_{i 2}\right)}, \quad H_{r}(s)=\frac{k_{r}\left(1+s \tau_{r 1}\right)}{\left(1+s \tau_{r 2}\right)^{2}} .
\end{aligned}
$$

The cross-over frequency can be varied by adjusting the corner frequencies, $\left(1 / \tau_{11}\right)$ and $\left(1 / \tau_{12}\right)$, of the integrators in $H_{t 1}$ and its dc gain $\left(k_{1}\right)$. Stability at the unity gain frequency is obtained by adjusting the corner frequency of the differentiator in $H_{2}$.

Initially, only the reaction mass was locally damped (i.e., $k_{i}=0$ ). Using the feedback parameters listed above, the cross-over frequency is about $0.15 \mathrm{~Hz}$ (about a factor of 4 below the lowest normal mode), and the system was stable with well-damped $(Q ' s \sim 2-10)$ normal modes. The model indicates that this system would have been stable without the local damping providing the $Q$ 's of the pendulums were $<3 \times 10^{2}$. In a high-sensitivity interferometer, however, where the pendulum $Q$ 's must be $>\sim 10^{8}$, this relatively low-cross-over-frequency systern would not have been stable in the absence of reaction mass damping.

As the cross-over frequency was increased to $0.6 \mathrm{~Hz}$ (just above the lowest normal mode), by decreasing the integrator time constants to $\tau_{11}=6.7 \times 10^{4} \mathrm{~s}$ and $\tau_{12}=0.47$ $\mathrm{s}$, the prototype system became unstable and stability could only be reattained by using both reaction and intermediate mass damping. This behavior agrees well with the predictions of the model. Further, the model predicts that the best damping of the normal modes will occur for $k_{r} \approx 1$ $\times 10^{3} \mathrm{~N} / \mathrm{m}$ and $k_{i} \approx 1.6 \times 10^{3} \mathrm{~N} / \mathrm{m}$. These values are similar to those found experimentally. Note that this system would be stable for arbitrarily high pendulum $Q$ 's. 


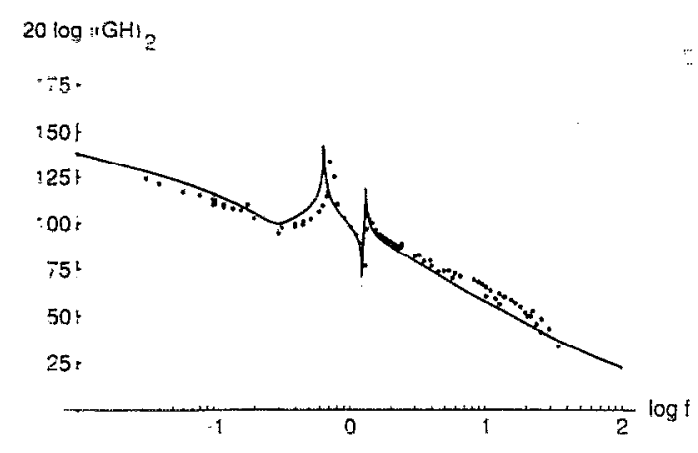

FIG. 7. Graph of a measured and predicted loop gain of the servo controlled, balanced two-stage pendulum.

The predicted rms closed-loop motion of the test mass is $X_{2 \mathrm{~ms}} \approx 1.4 \times 10^{-12} \mathrm{~m}$. In the proposed high-sensitivity interferometers, such a motion would ensure that only modest intensity stabilization would be required to reduce the sensitivity limit due to laser intensity noise ${ }^{1}$ below that due to shot noise. To further reduce the rms motion, one would need to increase the low-frequency gain and thus the servo bandwidth. This may not be possible, however, as parasitic high-frequency mechanical resonances might cause the servo to be unstable.

The open-loop gain was measured for a system having $\tau_{11}=1.4 \times 10^{5} \mathrm{~s}, \tau_{12}=1 \mathrm{~s}$, and $k_{r}=1 \times 10^{3} \mathrm{~N} / \mathrm{m}$ (cross-over frequency $\sim 0.3 \mathrm{~Hz}$ ). This system is in principle unstable if the pendulums have high $Q$ 's but was stable in practice due to the relatively low $Q$ 's of the pendulums in the prototype system. The loop gain was measured, as indicated earlier, by applying sine-wave signals of known amplitude and frequency to the PZT on which the reference mirror was mounted, and comparing the closed-loop displacement with the applied displacement. Applied displacements of several microns were used to allow measurements to be made at adequate signal-to-noise ratio. The measurements and the predicted open-loop gain are shown in Fig. 7. The predicted gain was calculated assuming $Q_{1}=200$, but it should be noted that the shape of this curve is relatively insensitive to the value of $Q_{1}$, and thus the agreement between experiment and theory is remarkably good.

\section{DISCUSSION}

We have experimentally demonstrated the use of a split feedback servo system for the control of the bottom mass in a balanced two-stage pendulum vibration isolation system. The importance of local damping of the intermediate and reaction masses in stabilizing the servo, particularly for high- $Q$ pendulum suspensions, has been highlighted. We have also presented a block diagram which accurately describes this multiple input, multiple output servo system, and could be used to determine the effect of noise added by the servo electronics.

While this article has only been concerned with the damping and control of the simple-pendulum modes of the suspension, we believe these techniques could also be used to ensure that the split-feedback remained stable in the presence of other high- $Q$ modes which might couple into the axial motion of the test mass.

\section{ACKNOWLEDGMENTS}

This research was supported by the SERC and the University of Glasgow. We would also like to thank fellow group members for useful discussions.

\section{APPENDIX}

The solution of the coupled equations of motion of the masses in a servo-controlled balanced two-stage pendulum will be outlined in this Appendix. The definition of various variables used in the body of the paper, e.g., $G_{i j}$ and $T_{i j}$, will also be given here.

The Laplace transformed equations of motion of the two-stage balanced pendulum shown in Fig. 2 are

$$
\left(\begin{array}{lll}
e_{11} & e_{12} & e_{13} \\
e_{21} & e_{22} & e_{23} \\
e_{31} & e_{32} & e_{33}
\end{array}\right)\left(\begin{array}{l}
X_{1} \\
X_{2} \\
X_{3}
\end{array}\right)=\left(\begin{array}{c}
F_{s}+F_{1} \\
F_{2} \\
-F_{2}+F_{3}
\end{array}\right)
$$

where

$$
\begin{aligned}
e_{11}= & m_{1} s^{2}+M \omega_{1}^{2}+m_{2} \omega_{2}^{2}+m_{3} \omega_{3}^{2}+m_{1} \omega_{1} s / Q_{1} \\
& +m_{2} \omega_{2} s / Q_{2}+m_{3} \omega_{3} s / Q_{3}, \\
e_{12}= & -m_{2} \omega_{2}^{2}-m_{2} \omega_{2} s / Q_{2}, \\
e_{13}= & -m_{3} \omega_{3}^{2}-m_{3} \omega_{3} s / Q_{3}, \\
e_{21}= & -m_{2} \omega_{2}^{2}-m_{2} \omega_{2} s / Q_{2}, \\
e_{22}= & m_{2} s^{2}+m_{2} \omega_{2}^{2}+m_{2} \omega_{2} s / Q_{2}, \quad e_{23}=0, \\
e_{31}= & -m_{3} \omega_{3}^{2}-m_{3} \omega_{3} s / Q_{3}, \quad e_{32}=0, \\
e_{33}= & m_{3} s^{2}+m_{3} \omega_{3}^{2}+m_{3} \omega_{3} s / Q_{3}, \\
M= & m_{1}+m_{2}+m_{3}, \quad F_{s}=\left(M \omega_{1}^{2}+m_{1} \omega_{1} s / Q_{1}\right) X_{s},
\end{aligned}
$$

$X_{1(2,3)}$ is the displacement of the intermediate (test, reaction) mass, $F_{1(3)}$ is the force applied to the intermediate (reaction) mass by its local damping actuators, $F_{2}$ is the force applied between the test and reaction masses, $X_{s}$ is the seismic displacement, and $m_{i}, \omega_{i}$, and $Q_{i}$ are the masses, uncoupled frequencies, and $Q$ 's of the pendula. The openloop (OL) response is thus given by 


$$
\begin{aligned}
& X_{1 \mathrm{OL}}=\frac{e_{22} e_{33}\left(F_{s}+F_{1}\right)+\left(e_{13} e_{22}-e_{12} e_{33}\right) F_{2}-e_{13} e_{22} F_{3}}{\operatorname{det}_{n f}}, \\
& X_{2 \mathrm{OL}}=\frac{-e_{21} e_{33}\left(F_{s}+F_{1}\right)+\left(e_{11} e_{33}-e_{13} e_{21}-e_{13} e_{31}\right) F_{2}+e_{13} e_{21} F_{3}}{\operatorname{det}_{n f}}, \\
& X_{3 \mathrm{OL}}=\frac{-e_{22} e_{31}\left(F_{s}+F_{1}\right)-\left(e_{11} e_{22}-e_{12} e_{21}-e_{12} e_{31}\right) F_{2}+\left(e_{11} e_{22}-e_{12} e_{21}\right) F_{3}}{\operatorname{det}_{n f}},
\end{aligned}
$$

where the determinant of the coefficient matrix, $[e]$, with no feedback, is given by

$$
\operatorname{det}_{n f}=e_{11} e_{22} e_{33}-e_{12} e_{21} e_{33}-e_{13} e_{22} e_{31} .
$$

Defining the mechanical responses, $G_{i j}$, by $G_{i j} \equiv X_{j \mathrm{OL}} / F_{i}$, where $F_{k}=0$ for $k \neq i$ and $F_{s}=0$, yields

$$
\begin{aligned}
& G_{12}=-e_{21} e_{33} / \operatorname{det}_{n f}, \\
& G_{22}=\left(e_{11} e_{33}-e_{13} e_{21}-e_{13} e_{31}\right) / \operatorname{det}_{n f}, \\
& G_{32}=e_{13} e_{21} / \operatorname{det}_{n f} .
\end{aligned}
$$

To determine the closed-loop (CL) transfer functions let $F_{1}=-H_{i} X_{1}-H_{t 1} X_{2}, F_{2}=-H_{i 2} X_{2}$, and $F_{3}=-H_{r} X_{3}$. The closed-loop response is then given by

$$
\begin{aligned}
& X_{1 \mathrm{CL}}=\frac{\left(e_{22}+H_{\imath 2}\right)\left(e_{33}+H_{r}\right) F_{s}}{\operatorname{det}_{f b}}, \\
& X_{2 \mathrm{CL}}=\frac{-e_{21}\left(e_{33}+H_{r}\right) F_{s}}{\operatorname{det}_{f b}}, \\
& X_{3 \mathrm{CL}}=\frac{-\left(e_{22} e_{31}+H_{t 2}\left(e_{21}+e_{31}\right)\right) F_{s}}{\operatorname{det}_{f b}},
\end{aligned}
$$

where the determinant of the coefficient matrix, $[e]$, with feed $b$ ack, is given by

$$
\begin{aligned}
\operatorname{det}_{f b}= & \left(e_{11}+H_{i}\right)\left(e_{22}+H_{t 2}\right)\left(e_{33}+H_{r}\right) \\
& -\left(e_{12}+H_{t 1}\right) e_{21}\left(e_{33}+H_{r}\right)-H_{t 2} e_{13} e_{21} \\
& -e_{13}\left(e_{22}+H_{t 2}\right) e_{31} .
\end{aligned}
$$

The coefficients which relate the closed-loop motion of the intermediate and reaction masses to that of the test mass are thus given by

$$
\begin{aligned}
& T_{21} \equiv X_{1 \mathrm{CL}} / X_{2 \mathrm{CL}}=-\left(e_{22}+H_{22}\right) / e_{21} \\
& T_{23} \equiv \frac{X_{3 \mathrm{CL}}}{X_{2 \mathrm{CL}}}=\frac{e_{22} e_{31}+H_{22}\left(e_{21}+e_{31}\right)}{e_{21}\left(e_{33}+H_{r}\right)} .
\end{aligned}
$$

'W. Winkler, in The Detection of Gravitatonal Waves, edited by D. G. Blair (Cambridge U. P., Cambridge 1991), p. 269; R. W. P. Drever, ibid. p. 306; J. Hough et al., ibid., p. 329.

${ }^{2}$ N. A. Robertson, ibid., p. 353.

${ }^{3} \mathrm{~J}$. Hough et al., "Proposal for a Joint German-British Interferometric Gravity Wave Detector" (Max-Planck-Institut fur Quantenoptik MPQ147, 1989); K. Danzmann et al., in Springer Lecture Notes in Physics (in press).

${ }^{4}$ R. E. Vogt, R. W. P. Drever, F. J. Raab, K. S. Thorne, and R. Weiss, A Laser Interferometer Gravitational-Wave Observatory (LIGO) (California Institute of Technology, CA, 1989)

${ }^{5}$ A. Giazotto, A. Brillet et al., The VIRGO Project (Instituto Nazionale di Fisica Nucleare, Sezione di Pisa, 1990).

${ }^{6}$ C. A. Cantley, J. Hough, N. A. Robertson, and R. J. S. Greenhalgh, Rev. Sci. Instrum. 63, 2210 (1992).

${ }^{7}$ N. A. Robertson et al., in Proceedings of the Fifh Marcel Grossmann Meeting on General Relativity, edited by D. G. Blair and M. J. Buckingham, (World Scientific, Singapore, 1989), p. 1775.

${ }^{8}$ C. A. Cantley, Ph.D. thesis, University of Glasgow (1991).

${ }^{9}$ M. Stephens, P. Saulson, and J. Kovalik, Rev. Sci. Instrum. 62, 924 (1991).

${ }^{10}$ D. Shoemaker, R. Schilling, L. Schnupp, W. Winkler, K. Maischberger, and A. Rudiger, Phys. Rev. D 38, 423 (1988).

${ }^{11}$ J. E. Faller, P. L. Bender, and R. T. Stebbins, "Low Frequency Isolation Systems for Gravitational Wave Interferometers," Joint Institute for Laboratory Astrophysics, unpublished. 